\title{
TPS: A Time-Based Positioning Scheme for Outdoor Wireless Sensor Networks
}

\author{
Xiuzhen Cheng \& Andrew Thaeler \\ Computer Science \\ The George Washington University \\ Washington, DC 20052, USA \\ \{cheng, athaeler\}@gwu . edu
}

\author{
Guoliang Xue \\ Computer Science \& Engineering \\ Arizona State University \\ Tempe, AZ 85287, USA \\ xue@asu.edu
}

\author{
Dechang Chen \\ Uniformed Services University \\ of the Health Sciences \\ Bethesda, MD 20817, USA \\ đchen@usuhs.mil
}

\begin{abstract}
In this paper, we present a novel time-based positioning scheme (TPS) for efficient location discovery in outdoor sensor networks. TPS relies on TDoA (TimeDifference-of-Arrival) of RF signals measured locally at a sensor to detect range differences from the sensor to three base stations. These range differences are averaged over multiple beacon intervals before they are combined to estimate the sensor location through trilateration. A nice feature of this positioning scheme is that it is purely localized: sensors independently compute their positions. We present a statistical analysis of the performance of TPS in noisy environments. We also identify possible sources of position errors with suggested measures to mitigate them. Our scheme requires no time synchronization in the network and minimal extra hardware in sensor construction. TPS induces no communication overhead for sensors, as they listen to three beacon signals passively during each beacon interval. The computation overhead is low, as the location detection algorithm involves only simple algebraic operations over scalar values. TPS is not adversely affected by increasing network size or density and thus offers scalability. We conduct extensive simulations to test the performance of TPS when TDoA measurement errors are normally distributed or uniformly distributed. The obtained results show that TPS is an effective scheme for outdoor sensor self-positioning.
\end{abstract}

\section{INTRODUCTION}

It is anticipated that wireless sensor networks will extend our sensory capability to every corner of the world. Distributed networks of thousands of collaborative sensors promise long-lived and unattended systems for many monitoring, surveillance and control applications. In this paper, we are going to examine the location discovery problem in outdoor wireless sensor networks. We will propose a novel time-based positioning scheme, henceforth referred to as TPS, that allows sensors to effectively determine their positions.

Many applications of outdoor sensor networks require knowledge of physical sensor positions. For example, target detection and tracking is usually associated with location information [19]. Further, knowledge of sensor lo- cation can be used to facilitate network functions such as packet routing [6], [16], [20], and collaborative signal processing [11]. Sensor position can also serve as a unique node identifier, making it unnecessary for each sensor to have a unique ID assigned prior to its deployment [34].

However, location discovery in wireless sensor networks is very challenging. First, the positioning algorithm must be distributed and localized in order to scale well for large sensor networks. Second, the localization protocol must minimize communication and computation overhead for each sensor since nodes have very limited resources (power, CPU, memory, etc.). Third, the positioning functionality should not increase the cost and complexity of the sensor since an application may require thousands of sensors. Fourth, a location detection scheme should be robust. It should work with accuracy and precision in various environments, and should not depend on sensor to sensor connectivity in the network. The TPS positioning scheme proposed in this research is designed to meet these challenges.

The major contribution of this paper is twofold. First, we propose a time-based location detection scheme for outdoor sensor networks and demonstrate our algorithm by simulation. Second, we analyze the theoretical performance of our scheme in noisy environments and identify possible sources of error with measures to help mitigate them. We put very few restrictions on the network layout and propose a scheme suitable for general outdoor sensor networks. We rely on RF signal, which performs well compared to ultrasound, infrared, etc., in outdoor environments [29]. We measure the difference in arrival times (TDoA) of beacon signals. In previous research, Timeof-Arrival (ToA) has proven more useful than RSSI in location determination [32]. TPS does not need the specialized antennae generally required by an Angle of Arrival (AoA) positioning system. This time-based location detection scheme avoids the drawbacks of many existing systems for outdoor sensor location detection. Our sim- 
ulations show that TPS is potentially very effective and computationally efficient.

Compared to existing schemes proposed in the context of outdoor sensor networks, our scheme has the following characteristics and advantages:

- Time synchronization of all base stations and nodes is not required in TPS. Sensors measure the difference in signal arrival times using a local clock. Base stations schedule their transmissions based on receipt of other beacon transmissions and do not require synchronized clocks. Many existing location discovery systems for sensor networks require time synchronization among base stations [25], or between satellites and sensors [15]. Imperfect time synchronization can degrade the positioning accuracy.

- There are no requirements for an ultrasound receiver [8], [32], second radio [15] or specialized antennae [5], [23], [25] at base stations or sensors. Our scheme does not incur the complexity, power consumption and cost associated with these components. (TPS sensors do require the ability to measure the difference in signal arrival times with precision.)

- Our algorithm is not iterative and doesn't require a complicated refinement step as does [28], [31], [33]. We refine position estimates by averaging time difference measurements over several beacon intervals prior to calculating position. This is useful to mitigate the effects of momentary interference and fast fading. This averaging requires less computation than repeatedly solving linear system matrices, least squares or multilateration algorithms.

- TPS has low computation cost. Our location detection algorithm is based on simple algebraic operations on scalar values. On the other hand, multilateration based systems [15], [17], [32], [33] require matrix operations to optimize the objective functions (minimum mean square estimation or maximum likelihood estimation), which induces higher computation overhead at each sensor.

- Sensors listen passively and are not required to make radio transmissions. Base stations transmit all the beacon signals. This conserves sensor energy and reduces RF channel use. Connectivity based systems often require global flooding [26] or global connectivity information [35] to estimate range.

This paper is organized as follows. Section II presents current location discovery techniques for outdoor sensor networks. Section III presents the network model. Section IV proposes TPS, a time-based location detection scheme. Its theoretic performance analysis is given in Section V. Simulation results are reported in Section VI.
We conclude our paper in Section VII.

\section{AN OVERVIEW ON CURRENT LOCATION DETECTION TECHNIQUES FOR SENSOR NETWORKS}

\section{A. Sensor Location Detection Techniques}

The majority of current sensor location detection schemes contain two phases: (i) range or angle measurement between sensors and base stations; and (ii) calculations which transform these measurements in to a position estimate. Some schemes perform a refinement phase after generating an initial estimate. In this subsection, we are going to examine related location discovery techniques.

\section{Range Estimation and Angle Measurement}

Popular techniques for range estimation include Timeof-Arrival (ToA), Time-Difference-of-Arrival (TDoA), and Received-Signal-Strength-Indicator (RSSI). Angleof-Arrival (AoA) involves measurement of the angle at which a signal arrives at a base station or a sensor. If there is no direct communication between base stations and sensors, network connectivity can be used for range estimation [26].

ToA and TDOA measure the signal arrival time or the difference of arrival times and calculate distance based on transmission times and speeds. They can be applied to many different kinds of signals such as RF, acoustic, ultrasound, etc. ToA has a disadvantage compared to TDoA as processing delays and non-LOS propagation can introduce errors [5]. ToA also requires synchronization to accurately measure time-of-flight. RSSI computes distance based on transmitted and received power levels, and a radio propagation model. RSSI is mainly used with RF signals. Due to multipath fading in outdoor environments, range estimation with RSSI can be inaccurate [32]. AoA is an attractive method due to the simplicity of the subsequent calculations (triangulation). But AoA can be difficult to measure accurately if a sensor is surrounded by scattering objects [5]. Further, measuring AoA requires sensors or base stations to be equipped with directive antennaes or antennae arrays, which may be prohibitive due to cost and form factors. Our time-based location detection scheme computes range based on TDo $\Lambda$ with no requirement for time synchronization. We actually detect the range differences from a sensor to three base stations (one is termed the master base station).

If a sensor can not receive signals from enough base stations ( $\geq 2$ for AoA, $\geq 3$ for ToA, TDoA, and RSSI), none of the previous techniques will work. In this case, network connectivity can be exploited for range estimation [26], [31]. DV-hop, DV-distance, and Euclidean are three range detection methods in this category. In $D V$-hop [26], 
[31] base stations flood their positions to all nodes in the network. Sensors compute the minimum distance in hops to several base stations. Base stations compute an average distance per hop to other base stations. The base stations then flood this information to the whole network allowing nodes to calculate their positions, DV-distance [26] replaces hop counts with cumulative range estimates in meters estimated from RSSI. Both techniques provide coarse range estimation to base stations and both require the expensive global flooding to compute the shortest path. Euclidean [26] estimates a sensor's distance to a base station based on the distance to two of its neighbors, the distance between the neighbors, and the distance from the neighbors to the base station. The Euclidean algorithm uses basic trigonometry to calculate distance to the base station. Each sensor needs to execute the Euclidean algorithm twice for two pairs of neighboring sensors to unambiguously determine its range to any base station.

\section{Location Computation from Range or Angle Measure- ments}

Triangulation, trilateration, and multilateration are the three techniques for combining ranges and angles. Triangulation is the simplest. As in Fig. 1(i), if the angles ( $\alpha$ and $\beta$ ) to base stations $\mathrm{A}$ and $\mathrm{B}$ are known, the location of $S$ is where lines from $\mathrm{A}$ and $\mathrm{B}$ intersect. Thus for AoA, at least two base stations are required. Trilateration computes the intersection of three circles, as shown in Fig. 1(ii). If the range to each base station is not accurate, the three circles may not have a common intersection point leading to ambiguous solutions. $M u l-$ tilateration uses an objective function to minimize the difference between the estimated position and real position of a sensor. For example in Fig. 1(iii), we can use $\min \Sigma_{i}\left(D_{S i}-\widehat{D}_{S i}\right)^{2}$ to compute $(x, y)$ for $\mathrm{S}$, where $D_{S i}=\sqrt{\left(x-x_{i}\right)^{2}+\left(y-y_{i}\right)^{2}}, \widehat{D}_{S i}$ is the estimated range from $S$ to $i, i=A, B, C, D, E$. This technique can improve accuracy but involves higher computation overhead. For details on multilateration, we refer the readers to [32]. Both trilateration and multilateration require at least 3 base stations. TPS uses trilateration with range difference information. We compute a sensor's position and its range to the master base station at the same time.

\section{B. Existing Sensor Location Detection Schemes}

GPS is the most popular localization system but may not be desirable in a sensor network due to cost, form factor, energy consumption, and the requirement for a second radio. GPS-less localization techniques have been researched extensively. For example, Ref. [3] proposes

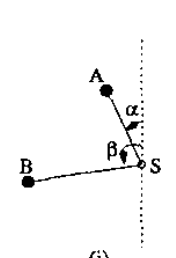

(i)

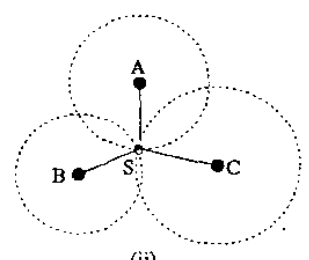

(ii)

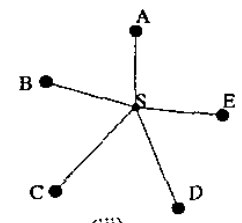

(iii)

Fig. 1. Range or angle combining techniques: (i) triangulation, (ii) trilateration, (iii) multilateration.

to use the centroid of multiple base stations to approximate the sensor location. In this subsection, we are going to overview in detail several works designed for outdoor sensor networks. For a taxonomy of location systems for ubiquitous computing we refer the readers to [12].

Ref. [32] proposes a TDoA based scheme (AHLOS) that requires base stations to transmit both ultrasound and $R F$ signals simultaneously. The RF signal is used for synchronization purposes. A sensor will measure the difference of the arrival times between the two signals and determine the range to the base station. Multilateration is applied to combine range estimates to generate location data. Testbed experiments demonstrate that AHLoS provides fine-grained localization capability. However, ultrasound transceivers can only cover a short range (several meters) and large numbers of base stations may be required to cover large areas. Other contributions by [32] include the introduction of iterative multilateration and collaborative multilateration. In iterative multilateration, a sensor becomes a base station after its position is determined. Whenever a sensor has range estimates to at least three base stations, multilateration is used to compute its position; otherwise, it continues to listen to beacon signals from base stations. If it is impossible for a sensor to find 3 base stations, collaborative multilateration can be used. In collaborative multilateration two or more sensors (which can be multiple hops apart) can form an overdetermined system of equations with a unique solution set. Feasible conditions for collaborative multilateration are further explored in [18], [33]. Ref. [17] compares the performance of different multilateration methods by simulation and proposes a new and fast iterative improvement algorithm to optimize location discovery. Ref. [8] designs and analyzes an acoustic ranging system for robotics ap- 
plications and embedded sensor technology. This paper examine methods to detect and eliminate various types of interference.

As mentioned earlier, AoA techniques require special antennae and may not perform well due to omnidirectional multipath reflections. To avoid requirements for directional antennae, Ref. [25] first transforms TDoA measurements in to AoA information and then applies triangulation to compute location. This scheme requires at least 3 base stations with synchronized rotating directional antennae. Non-zero antennae beam width and imperfect synchronization contribute to decrease system accuracy. A prototype navigation system based on AoA measurements for autonomous vehicles is presented in [23]. It estimates AoA by means of a set of optical sources and a rotating optical sensor. This system is not suitable for outdoor sensor networks due to its cost and complexity. Our scheme is similar to the one in Ref. [25] in that TPS measures TDoA at each sensor and has no additional special requirements for sensors. However, we do not use directional antenna in base stations and we do not require any kind of synchronization in the whole network.

The works mentioned above are all based on straightline range estimation to base stations. Ad Hoc Positioning system (APS) [26] first estimates ranges based on DV-hop, DV-distance, or Euclidean, and then applies trilateration to compute the location of each sensor. If enough base stations are available, location errors for APS with DVhop can be about $30 \%$ of radio range in a dense and regular topology. For sparse and irregular network topologies, the accuracy degrades to roughly the radio range. For DVdistance and Euclidean, the performance of APS also depends on the accuracy of the distance measured between neighboring sensors. Ref. [31] goes one step further: it refines location estimates computed by APS with DV-hop by using neighboring sensor position and distance estimates to help convergence to a better solution. To mitigate error propagation, a confidence weight from 0 to 1 is associated with each estimated position. With measured distance errors of 5\%, [31] produces an error of $33 \%$ of radio range on average for random graphs. Another work is [33], which uses DV-distance to compute range and Min-Max to compute position. To refine position estimates, [33] uses a computation tree. Ref. [18] compares [26], [31], and [33] in simulation.

Locating a subscriber in cellular networks or PCS systems has been well studied in literature [1], [5], [21]. The techniques involved produce coarse location granularity (tens or hundreds of meters), thus may not be suitable for outdoor sensor networks. Research on indoor or in-building localization is on-going and many interesting systems have been designed. Examples include Active Badge [36], Active Bat [10], RADAR [2], Cricket [27], SpotON [13], to name a few. Some of these systems require location surveys which are not possible with an airdeployed outdoor sensor network. For a brief overview on these systems, we refer the readers to [17]. Other interesting works in sensor networks include [7], [35], [30]. Ref. [7] proposes a positioning algorithm based on convex optimization. Ref. [35] describes a localization scheme based on multidimensional scaling. Both are centralized and both rely on connectivity information. Ref. [30] applies error detection and correction coding theory to location detection in emergency indoor sensor networks.

\section{NETWORK MODEL}

We assume that the sensors are deployed randomly over a 2-dimensional monitored area (on the ground). (However, Our proposed sensor positioning scheme can be easily extended to 3-dimensional space.) Each sensor has limited resources (battery, CPU, etc), and is equipped with an omni-directional antenna. Three base stations A, B, C, with known coordinates $\left(x_{a}, y_{a}\right),\left(x_{b}, y_{b}\right)$, and $\left(x_{c}, y_{c}\right)$, respectively, are placed beyond the boundary of the monitored area, as shown in Fig. 2. Let us assume A be the master base station. Assume the monitored area is enclosed within the angle $\angle B A C$. Let the unknown coordinates of a sensor be $(x, y)$, which will be determined by TPS. Each base station can reach all sensors in the monitored area. One restriction on the placement of these base stations is that they must be non-collinear, as otherwise, the sensor locations will be indistinguishable.

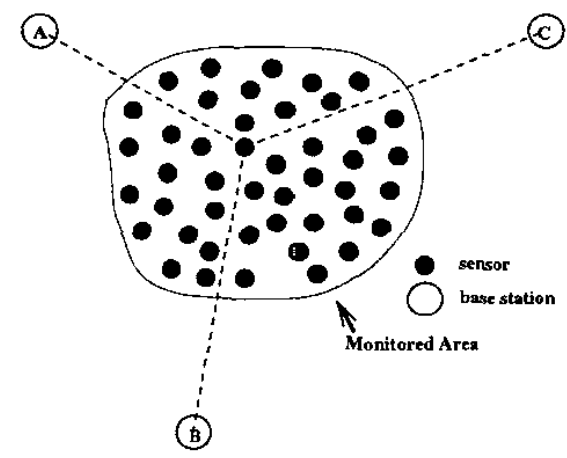

Fig. 2. An example sensor network.

Note that these base stations will transmit RF beacon signals periodically to assist each sensor with location discovery. They have long-term power supplies and can receive RF signals from each other. Note that there is no time synchronization among these three base stations. However, we require base stations to detect signal arrival 
times with precision and to accurately calculate total turnaround delay. This calculated turn-around delay consists of a random delay combined with known system transmission and reception delays.

Remark: If the monitored area is so large that 3 base stations can not cover the whole area completely, we can always divide the area into smaller subareas and place more base stations.

\section{TPS: A TIME-BASEd POSITIONING SCHEME}

In this section, we propose TPS, our time-based positioning scheme for outdoor wireless sensor networks. This scheme consists of two steps. The first step detects the time difference of signal arrival times from three base stations. We transform these time differences in to range differences from the sensor to the base stations. In the second step, we perform trilateration to transform these range estimates into coordinates.

\section{A. A Time-Based Location Detection Scheme}

Given the locations $\left(x_{a}, y_{a}\right),\left(x_{b}, y_{b}\right)$, and $\left(x_{c}, y_{c}\right)$ of base stations $\mathrm{A}, \mathrm{B}$, and $\mathrm{C}$, respectively, we are going to determine the location $(x, y)$ of sensor $S$, as shown in Fig. 3. Let $v$ be the speed of RF beacon signals from $\mathrm{A}, \mathrm{B}$, and C. Let $d_{a b}$ be the distance between base stations $\mathrm{A}$ and $\mathrm{B}$ and $d_{a c}$ be the distance between base stations $\mathrm{A}$ and $\mathrm{C}$. Thus $d_{a b}=\sqrt{\left(x_{a}-x_{b}\right)^{2}+\left(y_{a}-y_{b}\right)^{2}}$ and $d_{a c}=\sqrt{\left(x_{a}-x_{c}\right)^{2}+\left(y_{a}-y_{c}\right)^{2}}$. Let $d_{s a}, d_{s b}$, and $d_{s c}$ be the unknown distances from $S$ to $\mathrm{A}, \mathrm{B}$, and C respectively. Our time-based location detection scheme TPS consists of two steps.

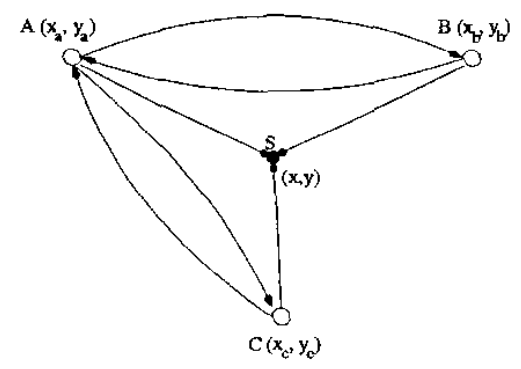

Fig. 3. Sensor $S$ will measure the TDoA of beacon signals from base stations A, B, and C locally. $S$ also will receive the turn-around delay information from B and C. B's transmission will start after it receives A's beacon signal, while C's transmission will start after it receives both A and B's beacon signals. This procedure will be repeated once every $T$ seconds.

Step 1: Range Detection.
Let $\mathrm{A}$ be the master base station, which will initiate a beacon signal every $T$ seconds. Each beacon interval begins when $\mathrm{A}$ transmits a beacon signal. Consider any beacon interval $i$, at times $t_{1}^{i}, t_{b}^{i}, t_{c}^{i}$, sensor $S$, base stations B and $C$ will all receive $A$ 's beacon signal respectively. At time $t_{b}^{i \prime}$, which is $\geq t_{b}^{i}$, B will reply to $\mathrm{A}$ with a beacon signal conveying information $t_{b}^{i \prime}-t_{b}^{i}=\Delta t_{b}^{i}$. This signal will reach $S$ at time $t_{2}^{i}$. After receiving beacon signals from both $\mathrm{A}$ and $\mathrm{B}$, at time $t_{c}^{i \prime}, \mathrm{C}$ will reply to $\mathrm{A}$ with a beacon signal conveying information $t_{c}^{i \prime}-t_{c}^{i}=\Delta t_{c}^{i}$. This signal will reach $S$ at time $t_{3}^{i}$. Based on triangle inequality, $t_{1}^{i}<t_{2}^{i}<t_{3}^{i}$. Let $\Delta t_{1}^{i}=t_{2}^{i}-t_{1}^{i}, \Delta t_{2}^{i}=t_{3}^{i}-t_{1}^{i}$, we obtain

$$
\begin{gathered}
d_{a b}+d_{s b}-d_{s a}+v \cdot \Delta t_{b}^{i}=v \cdot \Delta t_{1}^{i} \\
d_{a c}+d_{s c}-d_{s a}+v \cdot \Delta t_{c}^{i}=v \cdot \Delta t_{2}^{i}
\end{gathered}
$$

which gives

$$
\begin{aligned}
& d_{s b}=d_{s a}+v \cdot \Delta t_{1}^{i}-d_{a b}-v \cdot \Delta t_{b}^{i}=d_{s a}+k_{1}^{i} \\
& d_{s c}=d_{s a}+v \cdot \Delta t_{2}^{i}-d_{a c}-v \cdot \Delta t_{c}^{i}=d_{s a}+k_{2}^{i}
\end{aligned}
$$

where $d_{s a}, d_{s b}$ and $d_{s c}$ are positive real numbers and

$$
\begin{aligned}
k_{1}^{i} & =v \cdot \Delta t_{1}^{i}-v \cdot \Delta t_{b}^{i}-d_{a b} \\
k_{2}^{i} & =v \cdot \Delta t_{2}^{i}-v \cdot \Delta t_{c}^{i}-d_{a c} .
\end{aligned}
$$

Averaging $k_{1}^{i}$ and $k_{2}^{i}$ over $I$ intervals gives

$$
\begin{aligned}
& k_{1}=\frac{v}{I}\left[\sum_{i=1}^{I}\left(\Delta t_{1}^{i}-\Delta t_{b}^{i}\right)\right]-d_{a b}, \\
& k_{2}=\frac{v}{I}\left[\sum_{i=1}^{I}\left(\Delta t_{2}^{i}-\Delta t_{c}^{i}\right)\right]-d_{a c} .
\end{aligned}
$$

We are going to apply trilateration with $k_{1}$ and $k_{2}$ to compute coordinates $(x, y)$ for sensor $S$ in the next step.

Remarks: (i) All arrival times are measured locally. In other words, $t_{1}, t_{2}, t_{3}$ are measured based on sensor $S$ 's local timer; $t_{b}$ and $t_{b}^{\prime}$ are based on B's local timer and known system delays; while $t_{c}$ and $t_{c}^{\prime}$ are based on C's local timer and known system delays. There is no global synchronization. (ii) We require $\mathrm{A}$ to periodically initiate the beacon signal transmission for two reasons. First, averaging $k_{1}^{i}$ and $k_{2}^{i}$ over multiple beacon intervals helps to decrease the measurement error. The number of beacon intervals $I$ can be a trade-off between potential accuracy improvement and power consumption. Second, sensors may sleep to save energy; or they may be deployed at different times; or they may move during their lifetime. The periodic beacon signals from $\mathrm{A}$ and the reply signals from $\mathrm{B}$ and $\mathrm{C}$ can facilitate location discovery at any time. 
Step 2: Location Computation.

From Eqs. (3), (4), (7) and (8), we have

$$
\begin{aligned}
& d_{s b}=d_{s a}+k_{1}, \\
& d_{s c}=d_{s a}+k_{2} .
\end{aligned}
$$

Based on trilateration, we obtain three equations with three unknowns $x, y$, and $d_{s a}$, where $d_{s a}>0$.

$$
\begin{aligned}
& \left(x-x_{a}\right)^{2}+\left(y-y_{a}\right)^{2}=d_{s a}^{2} \\
& \left(x-x_{b}\right)^{2}+\left(y-y_{b}\right)^{2}=\left(d_{s a}+k_{1}\right)^{2} \\
& \left(x-x_{c}\right)^{2}+\left(y-y_{c}\right)^{2}=\left(d_{s a}+k_{2}\right)^{2}
\end{aligned}
$$

In the next Subsection, we will show how to compute $x, y$ and $d_{s a}$ efficiently. We will also give the conditions under which the solution set is unique.

\section{B. An Efficient Solution for Location Detection by Trilat- eration}

Without loss of generality, we assume the three base stations are located at $(0,0),\left(x_{1}, 0\right)$, and $\left(x_{2}, y_{2}\right)$, respectively, where $x_{1}>0, y_{2}>0$. In other words, $x_{a}=y_{a}=y_{b}=0, x_{b}=x_{1}, x_{c}=x_{2}$, and $y_{c}=y_{2}$. Let sensor $S$ be located at $(x, y)$. Note that we can always transform real positions to this coordinate system through rotation and translation. We want to compute the location for $S$.

From Eqs.(11),(12), and (13), we have

$$
\begin{gathered}
x^{2}+y^{2}=d_{s a}^{2}, \\
x^{2}-2 x x_{1}+x_{1}^{2}+y^{2}=d_{s a}^{2}+2 d_{s a} k_{1}+k_{1}^{2}, \\
x^{2}-2 x x_{2}+x_{2}^{2}+y^{2}-2 y y_{2}+y_{2}^{2} \\
=d_{s a}^{2}+2 d_{s a} k_{2}+k_{2}^{2} .
\end{gathered}
$$

Subtracting Eq. (14) from Eq. (15), we obtain

$$
2 x_{1} x=-2 k_{1} d_{s a}-k_{1}^{2}+x_{1}^{2} .
$$

Subtracting Eq. (14) from Eq. (16), we obtain

$$
2 x_{2} x+2 y_{2} y=-2 k_{2} d_{s a}-k_{2}^{2}+x_{2}^{2}+y_{2}^{2} .
$$

Multiplying Eq. (18) with $x_{1}$ and subtracting the product of Eq. (17) with $x_{2}$, we obtain

$$
\begin{aligned}
2 x_{1} y_{2} y= & \left(2 k_{1} x_{2}-2 k_{2} x_{1}\right) d_{s a}+k_{1}^{2} x_{2}-k_{2}^{2} x_{1} \\
& +x_{2}^{2} x_{1}+y_{2}^{2} x_{1}-x_{1}^{2} x_{2} .
\end{aligned}
$$

Since $x_{1}>0, y_{2}>0$, Eq. (17) and (19) can be rewritten as

$$
\begin{aligned}
x= & \frac{-2 k_{1} d_{s a}-k_{1}^{2}+x_{1}^{2}}{2 x_{1}} . \\
y= & \frac{\left(2 k_{1} x_{2}-2 k_{2} x_{1}\right) d_{s a}}{2 x_{1} y_{2}}+ \\
& \frac{k_{1}^{2} x_{2}-k_{2}^{2} x_{1}+x_{2}^{2} x_{1}+y_{2}^{2} x_{1}-x_{1}^{2} x_{2}}{2 x_{1} y_{2}} .
\end{aligned}
$$

Substituting Eqs. (20) and (21) into (14), we obtain

$$
\alpha d_{s a}^{2}+\beta d_{s a}+\gamma=0
$$

where

$$
\begin{aligned}
\alpha= & 4\left[k_{1}^{2} y_{2}^{2}+\left(k_{1} x_{2}-k_{2} x_{1}\right)^{2}-x_{1}^{2} y_{2}^{2}\right] \\
\beta= & 4\left[k_{1}\left(k_{1}^{2}-x_{1}^{2}\right) y_{2}^{2}+\left(k_{1} x_{2}-k_{2} x_{1}\right)\right. \\
& \left.\left(k_{1}^{2} x_{2}-k_{2}^{2} x_{1}+x_{2}^{2} x_{1}+y_{2}^{2} x_{1}-x_{1}^{2} x_{2}\right)\right], \\
\gamma= & \left(k_{1}^{2}-x_{1}^{2}\right)^{2} y_{2}^{2}+ \\
& \left(k_{1}^{2} x_{2}-k_{2}^{2} x_{1}+x_{2}^{2} x_{1}+y_{2}^{2} x_{1}-x_{1}^{2} x_{2}\right)^{2} .
\end{aligned}
$$

Theorem IV.1: Eq. (22) has a unique positive root for $d_{s a}$ if and only if one of the following three conditions holds.

1) $\alpha=0, \beta<0$, and $\gamma>0$;

2) $\alpha \gamma<0$;

3) $\alpha \beta<0, \gamma=\frac{\beta^{2}}{4 \alpha}$.

PROOF. We prove the theorem by case study. First, we consider the case where both $\alpha$ and $\beta$ are zero. In this case, (22) is either satisfied by all values of $d$ (when $\gamma=0$ ) or violated by every value of $d$ (when $\gamma \neq 0$ ).

Next we consider the case where $\alpha=0$ and $\beta \neq 0$. In this case, (22) has a unique root $d_{s a}=-\frac{\gamma}{\beta}$. Since $\gamma \geq 0,-\frac{\gamma}{\beta}$ is positive if and only if $\beta<0$ and $\gamma>0$. This corresponds to the first condition in the theorem.

In the rest of the proof, we will consider the cases where $\alpha \neq 0$. Consider the case where $\alpha \gamma<0$. This implies that $\gamma>0$ and $\alpha<0$. It also implies that $\beta^{2}-4 \alpha \gamma>\beta^{2}$. Therefore (22) has a unique positive root $d_{s a}=\frac{-\beta-\sqrt{\beta^{2}-4 \alpha \gamma}}{2 \alpha}$. This corresponds to the second condition in the theorem.

In the case where $4 \alpha \gamma>\beta^{2}$, the equation does not have any root in the real field.

In the case where $0<4 \alpha \gamma<\beta^{2}$, the equation has two roots $d_{s a}^{(1)}=\frac{-\beta+\sqrt{\beta^{2}-4 \alpha \gamma}}{2 \alpha}$ and $d_{s a}^{(2)}=\frac{-\beta-\sqrt{\beta^{2}-4 \alpha \gamma}}{2 \alpha}$ which have the same sign.

In the case where $4 \alpha \gamma=\beta^{2}$, the unique root of the equation is $d_{s a}=-\frac{\beta}{2 \alpha}$ which is positive if and only if $\beta<0$. This corresponds to the third condition in the theorem.

Next consider the case where $\gamma=0$. Note that $\gamma=0$ implies that $k_{1}^{2}=x_{1}^{2}$, which in turn implies that $k_{1}^{2} x_{2}$ $k_{2}^{2} x_{1}+x_{2}^{2} x_{1}+y_{2}^{2} x_{1}-x_{1}^{2} x_{2}=0$. Therefore $\gamma=0$ implies that $\beta=0$. In this case, the equation does not have a positive root. This completes the proof of the theorem.

Substituting the value of $d_{s a}$ into Eqs. (20) and (21), we will have the coordinates $x$ and $y$ for $S$.

In the above solution, we have used the square root function. Note that computing the square root $X$ of a positive number $N$ only requires a few iterations of Newton's 
method [24] in the form of $X:=0.5 *(X+N / X)$. Our simulation results show that four iterations are sufficient to produce accurate solutions.

Remarks: (i) Newton's method converges quadratically, thus solving trilateration functions can be done in a fast fashion. (ii) Compared to the other location detection methods in literature [17], [32], [33], [15], our scheme has an important advantage: we improve performance by refining in the first step - averaging time differences over multiple beacon intervals, which involves only simple algebraic operations. Refining through popular strategies like maximum likelihood or minimum mean square require more computation.

We note that data collected may have errors. When solving a system of linear equations such as those defined by (20) and (21), solutions are more accurate when the condition number (the condition number of a system of linear equations is the ratio of the largest eigenvalue over the smallest eigenvalue) is small [9]. We note that the condition number of the system of linear equations (20) and (21) is $\max \left\{\frac{x_{1}}{y_{2}}, \frac{y_{2}}{x_{1}}\right\}$. When designing the system, it is better to choose the locations of base stations so that the ratio $\frac{x_{1}}{y_{2}}$ is as close to 1 as possible. It is interesting to note that the value of $x_{2}$ does not affect the condition number of the system. In practice, we may choose the locations of the base stations so that they are sitting at the vertices of an equilateral triangle. In this case, the condition number will be 1.155 , which is very close to 1 , resulting in a very stable system.

To ensure the unique positive solution for $d_{s a}$, it suffices to have $\alpha \gamma<0$. From Eq. (25), $\gamma>0$. Thus the sufficient condition is reduced to $\alpha<0$. That is

$$
k_{1}^{2} y_{2}^{2}+\left(k_{1} x_{2}-k_{2} x_{1}\right)^{2}<x_{1}^{2} y_{2}^{2},
$$

which gives

$$
k_{1}^{2} y_{2}^{2}+k_{1}^{2} x_{2}^{2}+k_{2}^{2} x_{1}^{2}-2 k_{1} k_{2} x_{1} x_{2}<x_{1}^{2} y_{2}^{2} .
$$

In our simulation, this condition is satisfied in all cases where sensors are not in close proximity to or behind a base station. Near the base stations (interior to triangle), the solutions for $d_{s a}$ are both positive. If the position that corresponds to our measurements is interior to the triangle, $d_{s a}^{(2)}=\frac{-\beta-\sqrt{\beta^{2}-4 \alpha \gamma}}{2 \alpha}$ is the correct calculation.

\section{Theoretical PeRformance Analysis}

The trilateration equations (11), (12), and (13) determine coordinates $(x, y)$ for sensor $S$ based on the measured values $k_{1}$ and $k_{2}$. The inaccuracies of $k_{1}$ and $k_{2}$ cause sensor position errors. From Eqs. (7) and (8), $k_{1}$ and $k_{2}$ are the averaged results over $I$ beacon intervals, and based on the Central Limit Theorem, $k_{1}$ and $k_{2}$ are approximately normally distributed when $I$ is large. Therefore, without loss of generality, we may assume $k_{1}$ and $k_{2}$ are distributed according to $\mathcal{N}\left(\mu_{1}, \sigma_{1}^{2}\right)$ and $\mathcal{N}\left(\mu_{2}, \sigma_{2}^{2}\right)$, respectively. In this Section, we first give a statistical error analysis of sensor coordinate estimation. We then identify the major sources of errors affecting TPS's location detection accuracy based on the network model described in Section III.

\section{A. Theoretical Error Analysis}

To simplify the elaboration, we consider the case when base stations A, B, and C are located at $(0,0),(R, 0)$, and $(0, R)$, respectively. This base station placement corresponds to condition number 1 , which results in the most stable system. To further simplify the analysis, we consider the case when $S$ is equidistant to any base station. The general case can be analyzed similarly.

In our case, it is reasonable to assume $\mu_{1}=\mu_{2}=0$, and thus $k_{1} / R \approx 0, k_{2} / R \approx 0$. To facilitate our analysis, we further assume that $k_{1}$ and $k_{2}$ are independent. (In general, one can introduce correlation between $k_{1}$ and $k_{2}$.) Plugging $x_{1}=R, x_{2}=0$, and $y_{2}=R$ into Eqs. (23), (24), and (25), and simplifying the solution to Eq. (22) by approximating $k_{1}^{2} / R^{2}$ and $k_{2}^{2} / R^{2}$ with 0 , we end up with

$$
d_{s a} \approx \frac{\sqrt{2 R^{2}+2 k_{1} k_{2}}-\left(k_{1}+k_{2}\right)}{2} .
$$

Substituting the above into Eq. (20) yields

$$
x \approx \frac{R}{2}+\frac{k_{1} k_{2}}{2 R}-\frac{k_{1}}{2} \sqrt{2+\frac{2 k_{1} k_{2}}{R^{2}}} .
$$

Now replacing $\frac{k_{1} k_{2}}{R^{2}}=\frac{k_{1}}{R} \frac{k_{2}}{R}$ by 0 , we obtain

$$
x \approx \frac{R}{2}+\frac{k_{1} k_{2}}{2 R}-k_{1} \sqrt{\frac{1}{2}}=\frac{R}{2}+k_{1} k_{2}^{*}
$$

where $k_{2}^{*}=\frac{k_{2}}{2 R}-\sqrt{\frac{1}{2}}$. Similarly, from Eq. (21) we have

$$
y \approx \frac{R}{2}+\frac{k_{\mathrm{i}} k_{2}}{2 R}-k_{2} \sqrt{\frac{1}{2}}=\frac{R}{2}+k_{2} k_{1}^{*}
$$

where $k_{1}^{*}=\frac{k_{1}}{2 R}-\sqrt{\frac{1}{2}}$.

Since $(x, y)$ is used to estimate the location of $S$, the error in the estimation must be addressed. There are several ways to do this. The following is a common practice, where the variance of each variable is computed and the size of the variance or standard deviation is used as a measure of estimation error. 
As $k_{1}$ has a Gaussian distribution with mean $\mu_{1}$ and variance $\sigma_{1}^{2}$, and $k_{2}$ has a Gaussian distribution with mean $\mu_{2}$ and variance $\sigma_{2}^{2}$, the linear combination $k_{1}^{*}$ has a Gaussian distribution with mean $\frac{\mu_{1}}{2 R}-\sqrt{1 / 2}$ and variance $\frac{\sigma_{1}^{2}}{4 R^{2}}$, and $k_{2}^{*}$ has a Gaussian distribution with mean $\frac{\mu_{2}}{2 R}-\sqrt{1 / 2}$ and variance $\frac{\sigma_{2}^{2}}{4 R^{2}}$. Denote by $E(X)$ and $V(X)$ the mean and variance of a random variable $X$. We have, from Eq. (30),

$$
\begin{aligned}
V(x) & \approx V\left(k_{1} k_{2}^{*}\right) \\
& =E\left(k_{1} k_{2}^{*}\right)^{2}-\left[E\left(k_{1} k_{2}^{*}\right)\right]^{2} \\
& =E\left(k_{1}^{2}\left(k_{2}^{*}\right)^{2}\right)-\left[E\left(k_{1} k_{2}^{*}\right)\right]^{2} .
\end{aligned}
$$

By the independence between $k_{1}$ and $k_{2}$, we have

$$
\begin{aligned}
E\left(k_{1} k_{2}^{*}\right)= & E\left(k_{1}\right) E\left(k_{2}^{*}\right) \\
E\left(k_{1}^{2}\left(k_{2}^{*}\right)^{2}\right)= & E\left(k_{1}^{2}\right) E\left(k_{2} *^{2}\right) \\
= & {\left[V\left(k_{1}\right)+\left(E\left(k_{1}\right)\right)^{2}\right] } \\
& {\left[V\left(k_{2}^{*}\right)+\left(E\left(k_{2}^{*}\right)\right)^{2}\right] . }
\end{aligned}
$$

Therefore substitution gives

$$
\begin{aligned}
V(x) \approx & V\left(k_{1}\right)\left[E\left(k_{2}^{*}\right)\right]^{2}+V\left(k_{2}^{*}\right)\left[E\left(k_{1}\right)\right]^{2} \\
& +V\left(k_{1}\right) V\left(k_{2}^{*}\right) \\
= & \sigma_{1}^{2}\left(\frac{\mu_{2}}{2 R}-\sqrt{\frac{1}{2}}\right)^{2}+\frac{\sigma_{2}^{2}}{4 R^{2}} \mu_{1}^{2}+\sigma_{1}^{2} \frac{\sigma_{2}^{2}}{4 R^{2}} \\
= & \frac{\sigma_{1}^{2} \mu_{2}^{2}+\sigma_{2}^{2} \mu_{1}^{2}+\sigma_{1}^{2} \sigma_{2}^{2}}{4 R^{2}}+\sigma_{1}^{2}\left(\frac{1}{2}-\frac{\mu_{2}}{R} \sqrt{\frac{1}{2}}\right) .
\end{aligned}
$$

Since $\mu_{1}=\mu_{2}=0$, the above reduces to

$$
V(x) \approx \frac{\sigma_{1}^{2}}{2}+\frac{\sigma_{1}^{2} \sigma_{2}^{2}}{4 R^{2}}=\frac{\sigma_{1}^{2}}{2}\left(1+\frac{\sigma_{2}^{2}}{2 R^{2}}\right) .
$$

Similarly, we have

$$
\begin{aligned}
V(y) \approx & V\left(k_{1}^{*}\right)\left[E\left(k_{2}\right)\right]^{2}+V\left(k_{2}\right)\left[E\left(k_{1}^{*}\right)\right]^{2} \\
& +V\left(k_{1}^{*}\right) V\left(k_{2}\right) \\
= & \frac{\sigma_{1}^{2} \mu_{2}^{2}+\sigma_{2}^{2} \mu_{1}^{2}+\sigma_{1}^{2} \sigma_{2}^{2}}{4 R^{2}}+\sigma_{2}^{2}\left(\frac{1}{2}-\frac{\mu_{1}}{R} \sqrt{\frac{1}{2}}\right) \\
\approx & \frac{\sigma_{2}^{2}}{2}+\frac{\sigma_{1}^{2} \sigma_{2}^{2}}{4 R^{2}}=\frac{\sigma_{2}^{2}}{2}\left(1+\frac{\sigma_{1}^{2}}{2 R^{2}}\right) .
\end{aligned}
$$

From the above analysis, we have the following observations. First, the variance of both $x$ and $y$ depend on the variances of $k_{1}$ and $k_{2}$. Second, the variance of $k_{1}$ contributes more to that of $x$ than the variance of $k_{2}$; And the variance of $k_{2}$ contributes more to that of $y$ than the variance of $k_{1}$. Third, when $R$ is large, $V(x) \approx \sigma_{1}^{2} / 2$,
$V(y) \approx \sigma_{2}^{2} / 2$, showing that the variance of $x$ is dependent on that of $k_{1}$ while the variance of $y$ is dependent on that of $k_{2}$. Fourth, if $\sigma_{1}^{2}=\sigma_{2}^{2}$, the variances of $x$ and $y$ can be treated the same in practice.

We note that the above discussion is based only on the first two moments of the random variables $k_{1}$ and $k_{2}$. We have not taken advantage of the normality assumption of these two variables. In fact, with additional normality assumption on $k_{1}$ and $k_{2}$, we can obtain approximations to the distributions of $x$ and $y$. For example, since $k_{1}$ and $k_{2}$ are independent, the CDF $P(x \leq \alpha)$ of $x$ can be approximated by (for any real number $\alpha$ ),

$$
\iint_{\xi \eta \leq \alpha-R / 2} \frac{R}{\pi \sigma_{1} \sigma_{2}} \exp \left\{-\frac{1}{2} f(\xi, \eta)\right\} d \xi d \eta,
$$

where

$$
\begin{aligned}
f(\xi, \eta) & =\left(\frac{\xi-\mu_{1}}{\sigma_{1}}\right)^{2}+\left(\frac{2 R \eta-\mu_{2}+\sqrt{2 R^{2}}}{\sigma_{2}}\right)^{2} \\
& =\left(\frac{\xi}{\sigma_{1}}\right)^{2}+R^{2}\left(\frac{2 \eta+\sqrt{2}}{\sigma_{2}}\right)^{2} \\
& =\left(\frac{\xi}{\sigma_{1}}\right)^{2}+2 R^{2}\left(\frac{1+\sqrt{2} \eta}{\sigma_{2}}\right)^{2}
\end{aligned}
$$

The above results will help us to explain simulation results. In our simulation study (Section VI), we consider the cases when the errors of TDoA measurements at the sensor are normally distributed or uniformly distributed. The variance of TDOA measurements determines the variances of $k_{1}$ and $k_{2}$. Simulation results show that position error strongly depends on the variance of TDoA measurements.

\section{B. Sources of Errors}

There are three major sources of errors for our timebased location detection scheme: the receiver system delay, the wireless multipath fading channel, and the nonline-of-sight (NLOS) transmission. The receiver system delay is the time duration from which the signal hits the receiver antenna until the signal is decoded accurately by the receiver. This time delay is determined by the receiver electronics. Usually it is constant or varies in very small scale when the receiver and the channel is free from interference. This system delay can be predetermined and be used to calibrate the measurements. For example, base stations $\mathrm{B}$ and $\mathrm{C}$ can always eliminate the system delay from $\Delta t_{b}^{i}$ and $\Delta t_{c}^{i}$ before these values are conveyed to the sensors in their reply messages to A's beacon signal. Meanwhile, as $\Delta t_{1}^{i}$ and $\Delta t_{2}^{i}$ are measured by one sensor, 
the effect of receiver system delay may cancel out. Thus in our model, if base stations $B$ and $C$ can provide precise a priori information on receiver system time delay, their effect will be negligible.

The wireless multipath fading channel will greatly influence the location accuracy of any location detection system. Major factors influencing multipath fading [29] include multipath propagation, speed of the receiver, speed of the surrounding objects, and the transmission signal bandwidth. Multipath propagation refers to the fact that a signal transmitted from the sender can follow a multiple number of propagation paths to the receiving antenna. In our system, the performance is not affected by the speed of the receivers since all sensors and base stations are stationary. However, a moving tank in the surrounding area can cause interference.

There are two important characteristics of multipath signals. First, the multiple non-direct path signals will always arrive at the receiver antennae latter than the direct path signal, as they must travel a longer distance. Second, in LOS transmission model, non-direct multipath signals will normally be weaker than the direct path signal, as some signal power will be lost from scattering. If NLOS exists, the non-direct multipath signal may be stronger, as the direct path is hindered in some way. Based on these characteristics, scientists can always design more sensitive receivers to lock and track the direct path signal. For example, multipath signals using a pseudo-random code arriving at the receiver later than the direct path signal will have negligible effects on a high-resolution DS-BPSK receiver [4]. Our location detection scheme mitigates the effect of multipath fading by measuring TDoA over multiple beacon intervals. TDoA measurements have been very effective in fading channels, as many detrimental effects caused by multipath fading and processing delay can be cancelled [5].

Another factor related to wireless channels that causes location detection errors is NLOS transmission. To mitigate NLOS effects, base stations can be placed well above the surrounding objects such that there are line-of-sight transmission paths among all base stations and from base stations to sensors.

In the next section, we are going to study the performance of our TPS positioning scheme over fading channels. We will consider the inaccuracy of TDoA information measured at sensors only. The sources of errors under consideration include multipath fading and NLOS. Thus we are going to assume the TDoA measurements are either normally distributed or uniformly distributed. These assumptions are popular in literature for TDoA measurements [5], [17] in fading channels.

\section{Simulation}

Eqs. (11), (12), and (13) compute coordinates $x$ and $y$ for sensor $S$ based on $k_{1}$ and $k_{2}$, which are determined by the time-related values at the sensor $\left(\Delta t_{1}^{i}\right.$ and $\left.\Delta t_{2}^{i}\right)$ and base stations $\mathrm{B}\left(\Delta t_{b}^{i}\right)$ and $\mathrm{C}\left(\Delta t_{c}^{i}\right)$ over beacon interval $i$ (see Eqs. (7) and (8)). Thus the errors of $x$ and $y$ result from the measuring errors of $\Delta t_{1}^{i}, \Delta t_{2}^{i}, \Delta t_{b}^{i}$, and $\Delta t_{c}^{i}$. In this simulation, we assume the measuring errors of $\Delta t_{b}^{i}$ and $\Delta t_{c}^{i}$ are negligible. This is reasonable, as we can always take possible measures (see Subsection V-B). to decrease the measuring errors of base stations when the number of base stations is small (only 3 in our case). For example, base stations can be placed well above the surrounding objects to avoid multipath fading and NLOS transmission, and the system delay can be predetermined to calibrate the ToA measurements. (In this case, the sensor network resides in a 3-dimensional space. TPS needs to be modified accordingly.) On the other hand, Eqs. (7) and (8) tell us that the measuring error of $\Delta t_{b}^{i}\left(\Delta t_{c}^{i}\right)$ plays the same role as that of $\Delta t_{1}^{i}\left(\Delta t_{2}^{i}\right)$ in the computation of $k_{1}\left(k_{2}\right)$. Thus in our simulation study, we only consider the measuring errors of $\Delta t_{1}^{i}$ and $\Delta t_{2}^{i}$, which are termed TDoA measuring errors in the following description. We will study the influence of $I$ and $\sigma^{2}$ upon position error, where $I$ is the number of beacon intervals used to compute $k_{1}$ and $k_{2}, \sigma^{2}$ is the variance of the TDoA measuring error.

We use Matlab to code TPS. This tool provides procedures to generate normally distributed and uniformly distributed random numbers. Note that we do not use the sqrt function in Matlab. Instead, we use Newton's method described in Subsection IV-B. We found that 4 iterations generally yielded good results.

We first check the correctness of our scheme. In this simulation, no measuring errors are introduced. Base station $\mathrm{A}$ is the master base station. We randomly place sensors within the open area formed by the acute angle $\angle B A C$, as shown in Fig. 2. This area is termed feasible area. We found that sensors close to the base stations may have two computed locations: one within the feasible area, and one outside. This is because Eq. (22) generates two positive roots for $d_{s a}$. But if we throw away the solution that is outside the feasible area, we can always compute the location for each sensor correctly (uniquely). Thus in the following simulation, we only consider the solutions that are within the open area formed by $\angle B A C$. This is reasonable, as the base station locations and the master base station are known to each sensor.

Now we study the distribution of position errors over a 2D planar monitored area. Fig. 4 is drawn in 3D space which demonstrates position errors vs. the real positions 


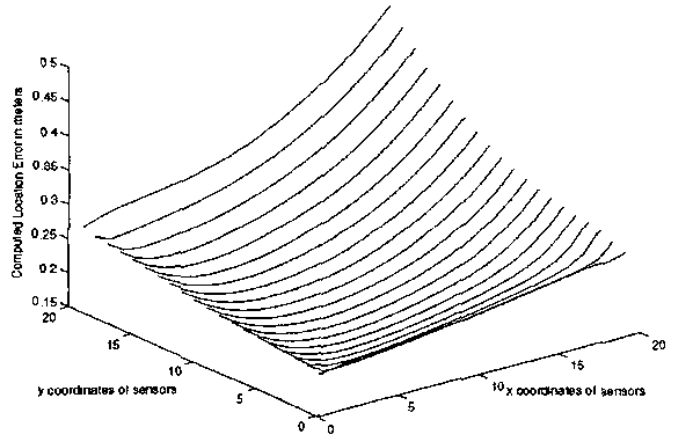

Fig. 4. Comparison of computed location errors by position. The sensors are placed at $19 \times 19$ grid points and $\sigma^{2}=0.05$. The base stations are located at $(0,0),(20,0)$ and $(0,20)$.

of sensors. In this simulation scenario, the three base stations are located at $(0,0),(20,0)$ and $(0,20)$. Sensors are placed at grid points $(i+0.5, j+0.5)$, where $i, j=0,1, \cdots, 19$. We average the sensor location results computed from our scheme over 10000 trials. For each trial, $I=4$. The measuring errors of $\Delta t_{1}^{i}$ and $\Delta t_{2}^{i}$ are normally distributed according to $\mathcal{N}(0,0.05)$. This corresponds to a TDoA deviation of \pm 0.22 unit. Note that we conduct extensive simulation over different $\sigma^{2}$ and achieve very similar results. Clearly this simulation shows that as the distance from a sensor to all three base stations become larger, the position error will become larger correspondingly. Also when the sensor is closer to any of the three base stations, the error becomes larger. We also observe that the sensor at location $(6.5,6.5)$, which is close to the intersection of the three angle bisectors of $\triangle A B C$, has the smallest position error and the sensors at its neighboring area also demonstrate quite low position errors. Interestingly, Refs. [25] and [3] provide similar results in their simulation study. Intuitively this is because the geometry of the intersection of the range circles is poor when the sensors are far away from any base station or when the sensors are close to any base station. From this analysis, we conclude that the position error is related to the placement of base stations. Careful studies will be conducted in the future as the results can be applied to guide the deployment of base stations for better performance. For these reasons, in the following simulation, we intentionally enforce an allowable shortest distance (1.0 unit) from any randomly generated sensor to any base station. This means the three base stations are placed some distance away from the boundary of the monitored area.

Next we consider the scenario when sensors are randomly deployed in a square region with lower-left corner $(1,1)$ and upper-right corner $(20,20)$. The three base sta- tions are still located at. $(0,0),(20,0)$, and $(0,20)$, respectively. We consider two error models; normal distribution according to $\mathcal{N}\left(0, \sigma^{2}\right)$ and uniform distribution over the range $[-b, b]$, which gives the variance $\sigma^{2}=b^{2} / 3$. For each variance value, we try 10000 random sensor positions. The averaged results are reported in Figs. 5 and 6. Note that we average over such a large number of sensor positions for each variance in order to take the whole monitored area into consideration. Also note that we report the simulation results when the number of beacon intervals $I$ used for position computation is chosen from the set $\{4,8,16,32,64,128\}$.

We obtain three observations from Figs. 5 and 6 . First, as $I$ increases, position error decreases. This is because averaging over larger number of beacon intervals to compute $k_{1}$ and $k_{2}$ can better smooth out the effects of measuring errors in TDoA measurements $\Delta t_{1}^{i}$ and $\Delta t_{2}^{i}$, thus produce improved result. A detailed theoretical explanation comes from Subsection V-A. As $I$ increases, $\sigma_{1}^{2}$ and $\sigma_{2}^{2}$ will decrease, and thus $V(x)$ and $V(y)$ will decrease. Then the errors from estimating the coordinates of sensors by $x$ and $y$ will decrease, implying that the position error will become smaller. Second, position error increases as variance $\sigma^{2}$ increases. This is reasonable as variance corresponds to the measuring error. Again, this can be well explained by Subsection V-A. In fact, if $\sigma^{2}$ increases, $\sigma_{1}^{2}$ and $\sigma_{2}^{2}$ will increase. Then $V(x)$ and $V(y)$ will increase so that the errors from estimating the coordinates of sensors by $x$ and $y$ will increase. Thus the larger the TDoA measuring error, the larger the position error. Third, for the same variance, position error is smaller when the TDoA measuring error is normally distributed. This is particularly true for small values of $I$. An explanation is given as follows. When $I$ is small, $k_{1}$ and $k_{2}$ are still normally distributed if TDoA measuring errors are normally distributed. However, for small $I, k_{1}$ and $k_{2}$ are not normal variables if the measuring errors are uniformly distributed. Following Subsection V-A, we can show that with fixed confidence, the predictive intervals of $x$ and $y$ for normally distributed measuring errors are narrower than those for uniformly distributed measuring errors. This shows that for normally distributed TDoA measuring errors, the position error is smaller. Note that shifting the square monitored area within $\angle B A C$, we obtain very similar results.

In the following, we report the simulation results when the base stations form a triangle such that $\angle B A C \leq 90^{\circ}$. In this simulation, three base stations are located at $(0,0)$, $\left(x_{1}, 0\right.$, and $\left(x_{2}, y_{2}\right)$, where $x_{1}, x_{2}$, and $y_{2}$ are randomly drawn from $[5,20] .100$ sensors are randomly placed within the overlapping area formed by $\angle B A C$ and the 


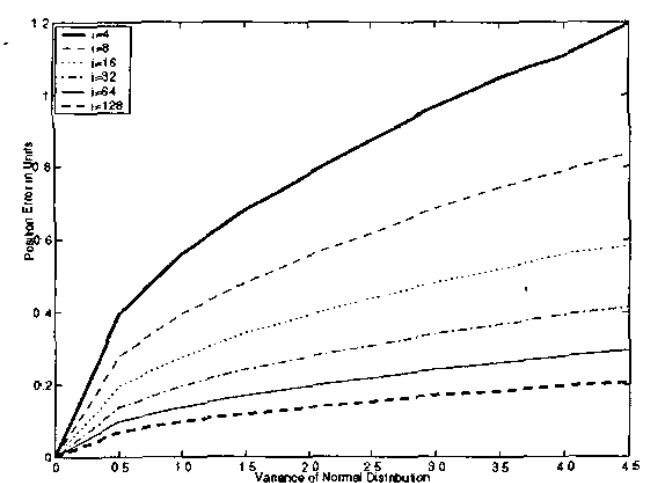

Fig. 5. Position error vs. variance $\sigma^{2}$. The TDoA measurements are normally distributed.

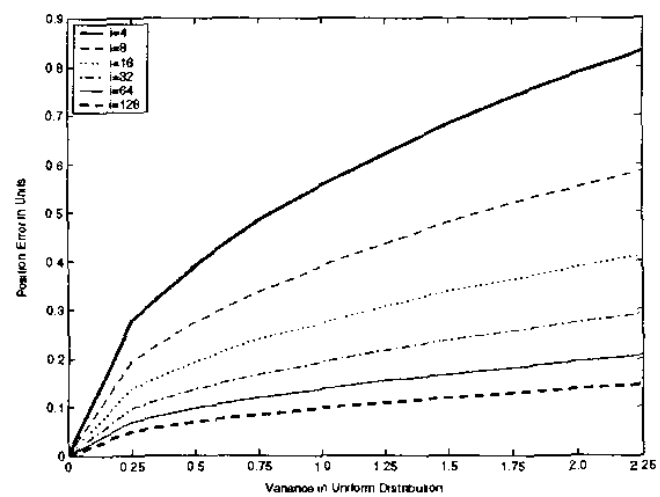

Fig. 6. Position error vs. variance $\sigma^{2}$. The TDoA measurements are uniformly distributed.

square with corners $(0,0)$ and $(20,20)$. We also throw away sensors whose distance to any base station is $<1.0$ unit, which means we only count sensors that are not too close to any base station. Fig. 7 reports the result when the TDoA measuring errors are normally distributed while Fig. 8 reports the result when the TDoA measuring errors are uniformly distributed. It is obvious that Figs. 7 and 8 are very similar to Figs. 5 and 6 , respectively. We obtain the same observations. However, for the same TDoA measuring error (same $\sigma^{2}$ ), we always achieve better performance in Figs. 7 and 8 . Thus $\angle B A C=90^{\circ}$ is not the optimal base station placement. This induces a network optimization problem, the base station placement prob$l e m$, for us to explore in the future.

\section{CONCLUSION}

In this paper, we presented TPS, a time-based positioning scheme for outdoor sensor networks. This scheme is superior to existing systems in many aspects such as com-

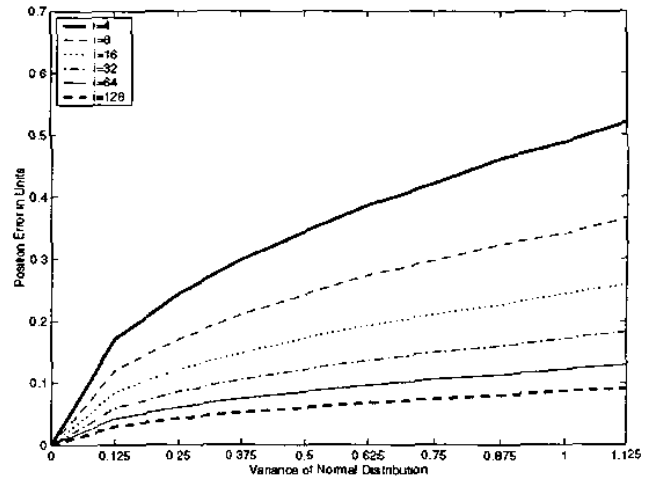

Fig. 7. Position error vs. variance $\sigma^{2}$. The TDoA measurements are normally distributed. Base stations are placed randomly within a fixed area.

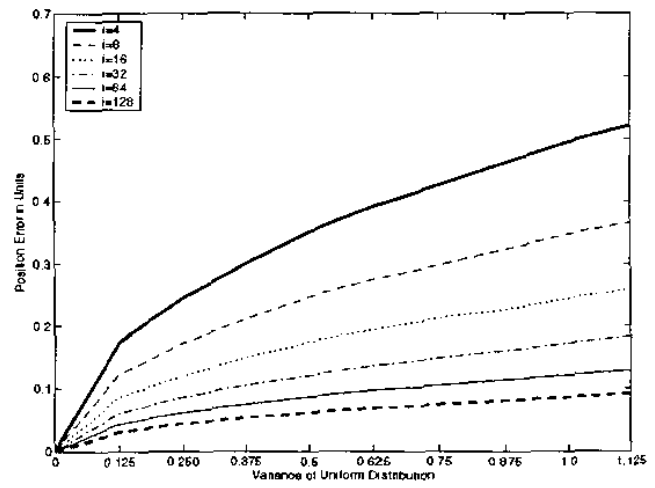

Fig. 8. Position error vs. variance $\sigma^{2}$. The TDoA measurements are uniformly distributed. Base stations are placed randomly within a fixed area.

putation overhead and scalability. To evaluate the performance of TPS, we conducted both theoretical analysis and simulations. Our scheme is simple and effective.

\section{ACKNOWLEDGMENT}

The research of $\mathrm{G}$. Xue was supported in part by ARO grant DAAD19-00-1-0377 and NSF ITR grant ANI0312635.

\section{REFERENCES}

[1] I.F. Akyildiz and W. Wang, A Dynamic Location Management Scheme for Next Generation Multi-tier PCS Systems, IEEE Transactions on Wireless Communications, Vol. 1, No. 1, pp. 178-190, 2002.

[2] P. Bahl and V.N. Padmanabhan, RADAR: an In-Building RFBased User Location and Tracking System, IEEE INFOCOM 2000, Vol. 2, pp. 775-784, 2000.

[3] N. Bulusu, J. Heidemann and D. Estrin, GPS-less Low Cost Outdoor Localization for Very Small Devices, IEEE Personal Communications Magazine, Special Issue on Networking the Physical World, August 2000. 
[4] J.J. Caffery, Jr and G. L. Stüber, Overview of Radiolocation in CDMA Cellular Systems, IEEE Communications Magazine, pp. 38-45, 1998.

[5] J.J. Caffery, Jr and G. L. Stüber, Subscriber Location in CDMA Cellular Networks, IEEE Transactions on Vehicular Technology, Vol. 47, No. 2, pp. 406-416, May 1998.

[6] S. De, C. Qiao, and H. Wu, Meshed Multipath Routing: An Efficient Strategy in Wireless Sensor Networks, Computer Networks, Special Issue on Wireless Sensor Networks, 2003.

[7] L. Doherty, K. Pister, and L. El Ghaoui, Convex Position Estimation in Wireless Sensor Networks, IEEE INFOCOM, 2001.

[8] L. Girod and D. Estrin, Robust Range Estimation Using Acoustic and Multimodal Sensing, IEEE/RSJ International Conference on Inteligent Robots and Systems, pp. 1312-1320, 2001.

[9] G.H. Golub and C.F. Van Loan, Matrix Computations, The Johns Hopkins University Press, 1990.

[10] A. Harter, A. Hopper, P. Steggles, A. Ward, and P. Webster, The Anatomy of a Context-Aware Application, MOBICOM, 1999.

[11] J. Heidemann and N. Bulusu, Using Geospatial Information in Sensor Networks, MOBICOM, 2000.

[12] J. Hightower and G. Borriello, Location Systems for Ubiquitous Computing, IEEE Computer, Vol. 34, No. 8, pp. 57-66, 2001.

[13] J. Hightower, R. Want, and G. Borriello, SpotON: an Indoor 3d Location Sensing Technology Based on RF Signal Strength, $U W$ CSE 2000-02-02, Univ. Washington, Seattle, 2000.

[14] C. Intanagonwiwat, R. Govindan and D. Estrin, Directed Diffusion: a Scalable and Robust Communication Paradigm for Sensor Networks, MOBICOM, pp. 56-67, 2000

[15] E.D. Kaplan (Editor), Understanding GPS: Principles and Applications, Artech House Publishers, 1996.

[16] B. Karp and H. Kung, GPSR: Greedy Perimeter Stateless Routing for Wireless Networks, MOBICOM, pp. 243-254, 2000.

[17] F. Koushanfar, S. Slijepcevic, M. Potkonjak, and A. SangiovanniVincentelli, Location Discovery in Ad-Hoc Wireless Sensor Networks, in Ad Hoc Wireless Networking (editors X. Cheng, X. Huang and D.-Z. Du), pp. 137-173, 2003.

[18] K. Langendoen and N. Reijers, Distributed Localization in Wireless Sensor Networks: a Quantitative Comparison, unpublished.

[19] D. Li, K.D. Wong, Y.W. Hu, and A.M. Sayeed, Detection, Classification, and Tracking of Targets, IEEE Signal Processing Magazine, March 2002.

[20] J. Li, J. Jannotti, D.S.J. DeCouto, D.R. Karger, and R. Morris, A Scalable Location Service for Geographic Ad Hoc Routing, MOBICOM, 2000.

[21] W. Ma and Y. Fang, Two-Level Pointer Forwarding Strategy for Location Management in PCS Networks, IEEE Transactions on Mobile Computing, Vol. 1, No. 1, pp. 32-45, 2002.

[22] S. Madden, M. J. Franklin and J.M. Hellerstein and W. Hong, TAG: a Tiny Aggregation Service for Ad-Hoc Sensor Networks, OSDI 2002.

[23] C.D. McGillem and T.S. Rappaport, A Beacon Navigation Method for Autonomous Vehicles, IEEE Transactions on Vehicular Technology, Vol. 38, No. 3, pp. 132-139, 1989.

[24] MIT Books On Line, Square Roots by Newton's Method, http://www-mitpress.mit.edu/sicp/chapter1/node9.html.

[25] A. Nasipuri and K. Li, A Directionality Based Location Discovery Scheme for Wireless Sensor Networks, WSNA'02, pp. 105$111,2002$.

[26] D. Niculescu, and B. Nath, Ad-Hoc Positioning System (APS), IEEE GlobeCom. 2001.

[27] N.B. Priyantha, A. Chakraborty, and H. Balakrishnan, The Cricket Location-Support System, MOBICOM, pp. 32-43, 2000.
[28] A. Rao, S. Ratnasamy, C. Papadimitriou, S. Shenker, and I. Stoica, Geographic Routing Without Location Information MOBICOM, pp. 96-108, 2003.

[29] T.S. Rappaport, Wireless Communications: Principles and Practice, 2nd Edition, Prentice Hall, 2002.

[30] S. Ray, R. Ungrangsi, F. De Pellegrini, A. Trachtenberg, and D. Starobinski, Robust Location Detection in Emergency Sensor Networks, IEEE INFOCOM, 2003.

[31] C. Savarese, J. Rabaey, and K. Langendoen, Robust Positioning Algorithms for Distributed Ad-Hoc Wireless Sensor Networks, USENIX technical annual conference, Monterey, CA, pp. 317$328,2002$.

[32] A. Savvides, C.-C. Han and M.B. Srivastava, Dynamic FineGrained Localization in Ad-Hoc Networks of Sensors, MOBICOM 2001, pp. 166-179, 2001.

[33] A. Savvides, H. Park, and M. Srivastava, The Bits and Flops of the N-hop Multilateration Primitive for Node Localization Problems, WSNA'O2, Atlanta, GA, pp. 112-121, 2002.

[34] C. Schurgers, G. Kulkarni, and M.B. Srivastava, Distributed On-Demand Address Assignment in Wireless Sensor Networks, IEEE Transactions on Parallel and Distributed Systems, Vol. 13, No. 10, pp. 1056-1065, 2002.

[35] Y. Shang, W. Ruml, Y. Zhang, and M. Fromherz, Localization From Mere Connectivity. MOBIHOC, 2003.

[36] R. Want, A. Hopper, V. Falcao, and J. Gibbons, The Active Badge Location System, ACM Transactions on Information Systems, pp. $91-102,1992$. 